\title{
Investigations of closure processes: What source-monitoring judgments suggest about what is "closing"
}

\author{
MARY ANN FOLEY and HUGH J. FOLEY \\ Skidmore College, Saratoga Springs, New York \\ FRANCIS T. DURSO \\ University of Oklahoma, Norman, Oklahoma \\ and \\ N. KYLE SMITH \\ Ohio State University, Columbus, Ohio
}

\begin{abstract}
On many occasions, the perception of an object is based on incomplete information because portions of the object are occluded from view. Nevertheless, people are able to identify an occluded object, suggesting that the identification is mediated by the activation of completion processes which "fill in" the missing information. Although these completion processes are invoked as causal mechanisms for many phenomena, the processes mediating the "completion" have not been specified. The present experiments tested two potential explanations for closure processes. In each experiment, participants were presented with pictures, complete or incomplete in form, and, on a surprise source-monitoring task, they were then asked to remember whether pictures had been presented complete or incomplete in form. Whether viewing pictures of objects, faces, or camouflaged objects, participants showed a pronounced tendency to claim that incomplete pictures were actually presented complete in form. This bias was evident after the participants described functions for objects (Experiments 1, 1A, and 2), after they evaluated properties of faces (Experiment 4), and after they searched for objects hidden in a complex scene (Experiment 5). However, this bias was not evident after they simply named objects portrayed in pictures (Experiment 3). This bias is interpreted as evidence for the activation of implicit imaginal processing. The results of these experiments are informative in their contribution to our understanding of the bases of closure, an intuitively appealing notion frequently invoked to account for object identification, but, as yet, not fully understood.
\end{abstract}

Imaginal processing is evoked spontaneously in a variety of contexts. Anecdotally, people report experiencing images spontaneously while listening to friends narrate life events or while reading novels (Golden, Foley, Holtz, \& Lynde, 1994). In experimental analogs, after reading extended narrative accounts of an event sequence, for example, people are more likely to claim they "saw" pictorial details related to events they had only read about (e.g., Belli, Lindsay, Gales, \& McCarthy, 1994; Intraub \& Hoffman, 1992). Actually, evidence for the activation and memorial effects of spontaneous imaginal processes in the absence of pictorial information occurs in a variety of contexts in response to words or more extended narratives (e.g., Denis, 1982; Hunt \& Marschark, 1987; Johnson-

The authors thank Karen Holtz, Roz McCall, Anna Nelson, and Brenna Worrell for their help in data collection and analyses. The authors also acknowledge Darcy Burgund for her careful reading of earlier versions of this manuscript and for her many helpful conversations about completion processes. Correspondence should be addressed to M. A. Foley, Department of Psychology, Skidmore College, Saratoga Springs, NY12866 (e-mail: mfoley@skidmore.edu).
Laird, 1983). When this imaginal processing is evoked automatically, which might lead it to more closely resemble perceptual processing, both adults and children have particular trouble distinguishing between what they saw and what they experienced imaginally, sometimes leading them to falsely claim an imagined object was actually "seen" (Durso \& Johnson, 1980; Foley, Durso, Wilder, \& Friedman, 1991). Notably, this confusion between words and images is considerably reduced in conditions in which people deliberately generate images prompted by words (Durso \& Johnson, 1980; Foley et al., 1991). Confusions between the actual content of text and images prompted by that text are interpreted as evidence that readers construct representations containing visual details of what they have read, and then they become uncertain about whether the visual details they are remembering were actually seen in pictures or only represented in thought (Durso \& Johnson, 1980; Foley et al., 1991; Johnson-Laird, 1983).

The descriptive language used to account for people's ability to identify objects under conditions of partial viewing implies the activation of similar kinds of imaginal pro- 
cessing. For example, people often see incomplete objects because portions of the objects are occluded from view. Nevertheless, they are able to identify these incomplete objects with little difficulty. In such situations, people are described as "filling in" the "missing" visual information in their mind's eye, "closing" the missing lines to render visually incomplete information as "complete." These filling-in processes, referred to as closure processes, are proposed as the basis for object identification in the presence of incomplete visual information (e.g., Gollin, 1962; Hearst, 1991; Matlin \& Foley, 1997; Ramachandran, 1992; Snodgrass \& Feenan, 1990).

Picture-fragmentation tasks provide one laboratory analog for this viewing experience. In a prototypical picture-fragmentation study, individuals are shown a series of incomplete pictures of an object, increasingly more complete, until they are able to identify the object presented in the series. Typically, individuals identify an object quickly and accurately before a series is completed (e.g., Gollin, 1962; Leeper, 1935; Murray \& Kinnison, 1989; Snodgrass \& Feenan, 1990; Snodgrass \& Surprenaut, 1989; Thurstone, 1944). Similarly, when used as a prime in visual matching tasks, an occluded object is as effective as one completely in view, suggesting that closure processes may be activated under initial viewing conditions (Sekuler \& Palmer, 1992; Sekuler, Palmer, \& Flynn, 1994).

Findings such as these from picture-fragmentation and visual matching tasks lead investigators to conclude that the identification of incomplete objects is mediated by closure processes, or the filling in of information missing from a pictorial display (e.g., Gibson, 1966; Gollin, 1962; Sekuler \& Palmer, 1992; Snodgrass \& Feenan, 1990; Snodgrass \& Surprenaut, 1989). Although closure processes are invoked as causal mechanisms for many phenomena, no one has determined what is actually mediating the closure (Sekuler \& Palmer, 1992). The absence of the specification of closure processes may indeed be a reflection of the intuitive appeal of the notion of closure (Sekuler \& Palmer, 1992). In the series of experiments reported in this paper, we begin to explore the kinds of processes that might mediate closure, considering two possibilities.

Closure processes may involve the activation of imaginal processes that function to fill in missing information in a visual stimulus, allowing a person to experience an object as complete in form. Alternatively, closure processes may refer to those conditions in which there is sufficient information in a visual stimulus to allow for its identification. This second possibility emphasizes the importance of the availability of sufficient perceptual information in a visual stimulus for resolving an object's identification. Although the results of picture-fragmentation tasks are typically used to suggest that implicit imaginal processing mediates closure (e.g., Snodgrass \& Feenan, 1990), it is at least plausible that people can succeed at these tasks without the activation of closure processes. From this point of view, as the information in the incom- plete picture increases, an individual may be more likely to identify the object portrayed in the incomplete version because there is more information explicitly available to resolve the object's identification, not because the individual is better able to "close" the lines. The use of the term closure to capture these effects would then be a misnomer.

In the present experiments, we adapted a procedure used to study the occurrence and consequences for memory of the activation of implicit imaginal processing in the absence of pictorial information to the study of the potential activation of these processes in the presence of incomplete pictorial information. Evidence for the activation of implicit imaginal processing in the absence of pictorial information comes from the study of failures to distinguish between memories for perceptual information (e.g., pictures or words) and memories for images (in this case, images evoked by words), which are illustrations of source-monitoring failures (Durso \& Johnson, 1980; Foley et al., 1991; Johnson, Hashtroudi, \& Lindsay, 1993; Johnson \& Raye, 1981). For example, consider a task in which adults and children are presented with pictures and words, and they are asked to describe functions for the referents in each case. Both adults and children are more likely to claim that a word was presented as a picture rather than the reverse. This misattribution bias is greater following referent tasks, such as function-identification tasks (i.e., in which individuals are asked to identify objects' functions), than following explicit imagery tasks (i.e., in which individuals are asked to generate images of the referents for the pictures and words).

The source-monitoring framework suggests why these failures may occur. Within this framework, the effect on source-monitoring judgments of orienting tasks is an indication of the role of cognitive operations information. When individuals generate images in response to explicit requests to do so, cues associated with more explicit imagery, such as the cognitive operations associated with forming an image, reduce the confusion between pictures and words (Durso \& Johnson, 1980; Foley et al., 1991). In contrast, when identifying objects' functions, individuals generate images implicitly in response to words, and these images are later mistaken as perceptual memories (here, memories for pictures). The confusion presumably occurs because the imaginal processing is automatic and not accompanied by cues associated with the deliberate generation of images. The results of a number of other studies of source monitoring converge in their support for the idea that an image may be mistaken for a perception when the image is automatically elicited (e.g., Johnson, Foley, Suengas, \& Raye, 1988; Johnson et al., 1993; Johnson, Raye, Foley, \& Kim, 1982; Johnson, Raye, Wang, \& Taylor, 1979).

We adapted these procedures to the study of closure processes by looking to see if people were confused about the way in which they saw pictures. In all of the experiments in this series, the visual materials (objects or faces) were depicted from frontal perspectives, but half of the 
depictions were incomplete, in that one side of the picture was deleted. In four of the experiments, removal of the visual information was explicit, with the left or right side of the pictured objects deleted. In the final experiment in the series, incomplete depictions were created by occluding portions of those pictures with other familiar objects. After viewing the pictorial materials while completing some kind of referent task (e.g., describing functions in Experiments 1, 1A, and 2, naming objects in Experiment 3, rating faces in Experiments 4 and $4 \mathrm{~A}$, or searching for hidden objects in Experiment 5), people were surprised with a source-monitoring task. They were asked to remember whether each of several test pictures was one presented complete in form, one presented incomplete in form, or a new one. Our index of the activation of implicit imaginal processes was the extent to which individuals were confused about the way in which the pictures were presented. If they claimed that incomplete pictures were presented as complete, it would suggest that people are filling in the missing visual information when viewing incomplete pictures. First, we looked to see if there was evidence for the bias and then proceeded to test alternative interpretations of its basis.

\section{EXPERIMENT 1}

In one condition of Experiment 1, people were shown pictures of familiar objects, complete or incomplete in form, and they were asked to identify the function of each referent pictorially presented. Later, they were asked to remember whether each of a number of pictures was presented previously. If they responded positively, they were further asked to identify whether each picture was presented complete or incomplete in form. In a second condition, other people were shown pictures or words referring to the same familiar objects. These people were also asked to describe the function of objects depicted as pictures or words and, later, to report how the objects were presented originally. This second condition served as a referent point for comparison with previous findings using this procedure to study implicit imaginal processing (Durso \& Johnson, 1980; Foley et al., 1991).

\section{Method}

Participants. Twenty-four undergraduates attending Skidmore College volunteered to participate in this experiment and received extra course credit for their participation. Males and females were represented proportionally.

Materials. Ninety-six items were selected from the Snodgrass and Vanderwart (1980) norms, with half serving as targets and half as distractors on an unexpected memory test. The pictorial materials were equated for their familiarity and imagery agreement (defined as the extent to which a particular picture represents the image that first comes to mind when visualizing particular objects). Using Snodgrass and Vanderwart's norms (1980), the average ratings were 3.75 and 3.85 out of 5.0 for the familiarity and imagery agreement, respectively. During the first phase of the experiment, the items were presented individually on $5 \times 7$ in. index cards, at an 8 -sec rate, and in random order; two different presentation orders were used with equal frequency. The incomplete pictures were created by cutting each picture in half, and the frequency with which the left or right side of the pictures was removed was counterbalanced.

In the complete/incomplete picture condition, individuals were shown 48 targets, 24 pictures complete in form and 24 incomplete in form. In the picture/word condition, individuals were shown 48 targets as 24 pictures and 24 words. In each condition, the materials were counterbalanced for presentation mode so that each target occurred equally often in its complete or incomplete form or as a picture or word.

Procedure. Each person was tested individually by an experimenter blind to the purpose of the experiment. In both conditions, the participants were asked to describe something they might do with each object. They were told that their responses would help us norm materials to include in a developmental study. After a 5 -min retention interval, during which time the experimenter asked the participants to play a probability game, they were surprised with a source-monitoring test. The list of 96 test items was presented orally. In the complete/incomplete picture condition, the participants were asked whether an item was one they had seen before as a complete picture, an incomplete picture, or one they had not seen earlier in the session. In the picture/word condition, the participants were asked to indicate whether each item was one they had seen before as a picture, one they had seen as a word, or one they had not seen earlier in the session.

\section{Results}

Source monitoring. Responses on the source-monitoring test were assessed by computing a discrimination score--that is, the number of targets whose presentation mode was correctly identified (complete vs. incomplete, or picture vs. word) divided by those correctly recognized as old. For example, when materials were presented as complete or incomplete pictures, the discrimination score for complete pictures equaled the number of complete pictures correctly called "complete," divided by the number of complete pictures called either "complete" or "incomplete" indicating that the participants recognized them as old items. Similarly, the discrimination score for incomplete pictures refers to the number of incomplete pictures correctly labeled "incomplete" divided by the number labeled "incomplete" or "complete." Because the source-monitoring judgments were based on different types of target items in the two conditions, judgment condition was not included as a factor in the analyses of variance (ANOVA). Rather, each condition was analyzed separately. In the following two analyses, mode of presentation (pictures vs. words, whole vs. partial picture) was the independent variable tested, with an alpha level of .05 .

Complete/incomplete source-monitoring judgments. An ANOVA on discrimination performance for the participants classifying pictures as complete or incomplete in form showed a main effect for presentation mode. If people "complete" incomplete pictures in their mind's eye when describing objects' functions, then we would expect to observe a particular kind of confusion on the source-monitoring task. People should be more likely to claim that an incomplete picture was presented as complete rather than the reverse. Thus, a bias of this sort would lead to lower discrimination scores for incomplete pictures. This is, in fact, the pattern we observed: The participants were more likely to claim that a partial picture was 
presented previously in its complete form rather than the reverse, resulting in significantly lower discrimination scores for incomplete pictures $(M=0.82)$ than for complete pictures $(M=0.95)\left[F(1,11)=8.03, M S_{\mathrm{e}}=0.026\right.$, $p<.02]$.

Picture/word source-monitoring judgments. An analysis of the discrimination performance for those individuals making picture/word judgments also showed a main effect for presentation mode $\left[F(1,11)=7.93, M S_{\mathrm{e}}=\right.$ $0.029, p<.02]$, indicating that the participants were more likely to claim that a word was presented previously as a picture rather than the reverse. This error pattern produced lower discrimination scores for words $(M=0.85)$ than for pictures $(M=0.99)$, a pattern replicating previous findings (Durso \& Johnson, 1980; Foley et al., 1991).

Recognition. To show that the discrimination performance just reported was not simply an alternative measure of recognition, the source-monitoring test was rescored for proportion of hits. When the responses on the source-monitoring test were scored to calculate proportion of hits (i.e., the proportion of items recognized as old whether or not the participants correctly remembered their mode of presentation), no significant effects were observed for either condition. Similarly, the proportion of false positives did not vary with response types in either condition (i.e., falsely reporting "whole" or "partial" picture). Table 1 summarizes these two measures for both conditions. Thus, the bias reported in the discrimination data is not an index of poor memory for the incomplete versions of target items.

An alternative technique for assessing source-monitoring judgments provided by Batchelder and Reifer (1990) was also used, although this technique has come under criticism recently (Kinchla, 1994) because it departs from most approaches for assessing recognition performance. The effects were essentially the same as those reported above, largely because recognition performance was quite good. Recognition did not vary for complete and incomplete pictures, a situation in which the Batchelder and Reifer procedure for assessing sourcemonitoring judgments could potentially lead to different

Table 1

Overall Recognition Performance in Experiment 1 Whole/Part Picture Condition

Proportion Hits

Complete pictures

Incomplete pictures

.98

Proportion False Positives

Falsely reporting:

"Complete picture"

"Incomplete picture"

Picture/Word Condition

Proportion Hits

Pictures

.98

Words

Proportion False Positives

Falsely reporting

"Picture"

"Word"

.08 results, though not always (Batchelder and Reifer, 1990). In fact, when the Batchelder and Reifer (1990) procedure was applied to source-monitoring errors reported previously (Foley et al., 1991), there were no differences in the results in that case either.

Experiment 1A. Because Experiment 1 was the first use of a source-monitoring procedure to study closure processes, we conducted a second version of the complete/ incomplete picture condition $(n=10)$. We replicated the effects just reported for Experiment 1, finding a bias to claim that incomplete pictures were presented complete in form. Again, simple recognition of pictorial materials was quite good, and there were no effects for visual complexity on either discrimination or recognition measures.

\section{Discussion}

The discrimination data from Experiment 1 replicate previous findings showing that individuals are more likely to claim that a word had been presented previously as a picture rather than the reverse (Durso \& Johnson, 1980; Foley et al., 1991). The new finding was the observation of the tendency to claim that an incomplete picture was presented in its complete form (Experiments 1 and $1 \mathrm{~A}$ ). The results also showed no bias to classify new items in a particular way if they were misidentified as old (Experiments 1 and 1A), suggesting that the bias we observed to misclassify an old incomplete picture as complete in form is not due to a general response bias to say "complete." However, because the false-positive rate was quite low in Experiments 1 and 1A, the absence of a response bias to say "complete" may have been masked by a ceiling effect. More compelling evidence that the bias to report "complete" is not an indication of a general response bias is provided by a subsequent experiment in this series (Experiment 4).

Our preferred interpretation for this bias to claim that an incomplete picture was presented as complete is that the incomplete pictorial information evoked implicit imaginal processing, rendering the incomplete picture as "complete," and this imaginal filling-in process created the subsequent confusion about the way the pictures were presented. However, we should consider an alternative interpretation of these results. The opportunity for accumulating perceptual information could be greater for incomplete pictures if people inspect those pictures for a considerable length of time before describing objects' functions. In Experiment 2, we again asked participants to describe objects' functions, but, in this case, we also measured the time it took them to begin to describe the functions. The latency to respond was our index of the potential availability of perceptual information. Following the function task, participants were given a sourcemonitoring test, and they were asked to remember the way in which the pictures were presented.

From one point of view, perceptual information is potentially more available the longer the duration between the presentation of a picture and a person's report of a function of the object portrayed in that picture. If the availability of perceptual information is contributing to 
the bias we observed, then there should be a systematic relationship between source monitoring and the potential availability of perceptual information. Potential increases in the availability of perceptual information could induce source-monitoring errors (hence, lowering discrimination scores) if impressions of visual detail, one kind of perceptual information, falsely signal that an incomplete picture was presented as complete in form. We know that source-monitoring judgments are affected by sensory detail with this detail falsely signaling that a memory was perceptually derived (e.g., Johnson, Foley, Suengas, \& Raye, 1988; Johnson et al., 1982). Thus, if memory for a picture includes an impression of detail, a person might falsely conclude that an incomplete picture was presented as complete.

Research in other contexts leads to a different prediction about the relationship between source monitoring and latencies. Often when information is available for a longer period of time, subsequent responses (memory, decision making, problem solving) are more accurate. In the present context, this would lead us to expect a positive relationship between response time and source-monitoring performance. The important point, however, is that both predictions suggest that source-monitoring performance and latencies will be related systematically. We considered these possibilities in Experiment 2.

If the availability of perceptual information influences source monitoring, then the relative amount of visual detail in pictures should influence performance as well because visual detail is a type of perceptual information (Johnson et al., 1993). Therefore, in Experiment 2, we also manipulated an aspect of the pictures themselves to vary the impression of visual detail, one form of perceptual information. Visual detail was varied by presenting pic- tures that were either visually simple or visually complex in their detail.

\section{EXPERIMENT 2}

\section{Method}

Participants. Forty-eight undergraduates attending Union College were paid \$2 for their participation. Males and females were represented proportionally.

Materials. The materials comprised 96 pictures selected from the Snodgrass and Vanderwart's (1980) norms. These pictorial renderings varied in two ways: The line drawings were either simple or complex in their visual detail, and the versions that participants experienced either were analogous to the kinds they typically experienced when viewing pictures of this sort or were modified. For example, on the basis of the Snodgrass and Vanderwart (1980) norms, a picture of an envelope is naturally rendered as visually simple, whereas a picture of a butterfly is naturally experienced as visually complex. For these normative versions (referred to as natural renderings), the average complexity ratings for simple and complex renderings were 1.70 and 3.43 for visual detail. These materials were equated for familiarity $(M \mathrm{~s}=3.79$ and 3.69 , respectively) and for imagery agreement ratings $(M \mathrm{~s}=3.92$ and 3.80 , respectively). For modified versions, pictures that were originally simple in their visual detail were modified to be complex in form, and those that were originally complex in their visual detail were modified to be simple in form (in the present examples, a visually detailed picture of an envelope and a visually simple version of a butterfly). Examples are provided in Figure 1. These modified versions were comparable in their visual detail to natural versions of simple and complex pictures.

Specifically, of the 48 target items, 24 were presented in the original versions included in the Snodgrass and Vanderwart (1980) norms ( 12 visually simple and 12 visually complex). However, the remaining 24 target items were modified so that 12 pictures that were originally simple in their visual detail on the basis of the norms were modified to be complex in form, and 12 that were originally complex in their visual detail were modified to be simple in

\section{PICTURE VERSION EXPERIENCED DURING STUDY}

\section{VISUALLY \\ SIMPLE}

\section{TYPICALLY}

EXPERIENCED

SIMPLE

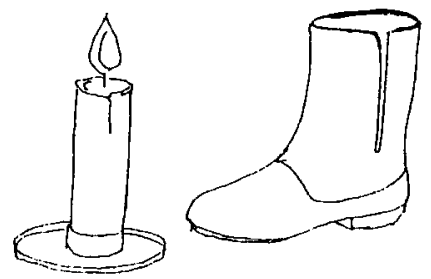

COMPLEX

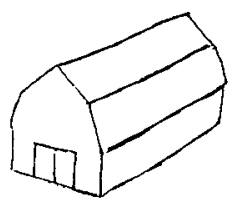

VISUALLY

COMPLEX 
form. Equal numbers of original and modified versions were presented complete (6) and incomplete (6) in form. Materials were counterbalanced, appearing equally often in each item-type category. In summary, there were three manipulations of the materials: completeness (complete vs. incomplete in form), natural rendering (simple vs. complex), and actual rendering (simple vs. complex).

Apparatus. A procedural change was also introduced in Experiment 2 in that materials were presented on a computer screen rather than on index cards. A Macintosh computer was used to control picture presentations and to record response times. The pictures were presented in the center of the monitor located directly in front of the individual.

Procedure. Each person, tested individually, was told that his or her task was to press any response key as soon a function for each object came to mind and to describe the function out loud. Times to respond were recorded automatically, and the experimenter recorded any naming errors (e.g., calling an ashtray a bowl). After a 5-min retention interval, during which time the participants worked on a number search task as a distractor, they were surprised with a source-monitoring test. A list of 96 test items was presented, and the participants were asked to record whether or not each item named an object they had seen before, and, if yes, whether it was presented as a complete or incomplete picture. Following each source decision, the participant also rated the confidence in his or her judgment using a 9-point rating scale.

\section{Results}

Source monitoring. As in Experiments 1 and $1 \mathrm{~A}$, source-monitoring ability was assessed with a discrimination score, the proportion based on the number of items whose presentation mode was correctly identified relative to those correctly recognized as old. For example, when materials were presented as whole pictures, the discrimination score for complete pictures refers to the number of complete pictures correctly called "complete" divided by the number of complete pictures called either "complete" or "incomplete," indicating that the participants recognized them as old items. Source-monitoring scores were computed for the four sets of complete pictures: typical rendering simple/actual rendering simple, typical rendering simple/actual rendering complex, typical rendering complex/actual rendering simple, and typical rendering complex/actual rendering complex. Four sets of comparable scores were computed for the corresponding sets of incomplete pictures.

An ANOVA on the source-monitoring scores included three factors: completeness (complete vs. incomplete in form), typical rendering (simple vs. complex in visual detail), and actual rendering (simple vs. complex). There were two significant effects. The interaction between natural rendering (simple vs. complex) and actual rendering (simple vs. complex) was significant $[F(1,45)=5.79$, $\left.M S_{\mathrm{e}}=0.01, p<.02\right]$. The main effect for natural rendering was also significant $\left[F(1,45)=10.40, M S_{\mathrm{e}}=0.01\right.$, $p<.003]$. The means are shown in Table 2. Post hoc tests showed that discrimination performance was lower for pictures of objects typically rendered as visually complex and actually rendered in that way during the experimental session $(M=0.85)$ than for each of the other three categories ( $M \mathrm{~s}$ ranged from .91 to .94 ). Post hoc tests also showed that there were no differences in source-
Table 2

Average Source-Monitoring Scores of Experiment 2 (Describing Objects' Functions)

\begin{tabular}{lcc}
\hline & \multicolumn{2}{c}{ Actual Rendering } \\
\cline { 2 - 3 } Natural Rendering & Visually Simple & Visually Complex \\
\hline Visually simple & .91 & .94 \\
Visually complex & .91 & .85 \\
\hline
\end{tabular}

monitoring discrimination scores for the other three picture categories (see Table 2).

To see why discrimination scores were lower for pictures usually experienced as visually complex and presented in that way in this experiment, we compared the source-monitoring scores for complete and incomplete pictures within this category. Post hoc tests showed that the participants were more likely to classify these incomplete complex pictures as complete in form than they were to classify these complete complex pictures as incomplete. Thus, discrimination scores were lower for incomplete pictures $(M=0.84)$ than for complete pictures $(M=0.90)$. The lower performance in this picture category was a result of greater confusion about incomplete pictures, which replicates the pattern reported in Experiment 1.

In a separate analysis on only those objects rendered in typical ways, as was the case in Experiments 1 and $1 \mathrm{~A}$, we replicate our findings: Incomplete pictures are misclassified as complete more often than the reverse. The average discrimination scores were 0.91 and 0.87 for complete and incomplete pictures (averaging across simple and complex detail). In sum, the same general pattern reported for Experiments 1 and $1 \mathrm{~A}$ was observed: Incomplete pictures were classified as complete more often than the reverse.

Latencies. As we mentioned earlier, if the availability of perceptual information could account for the sourcemonitoring discrimination scores, we might expect to find a decrease in source-monitoring performance with an increase in the availability of perceptual information (in Experiment 2, indexed by an increase in latency to respond). To see whether or not there was a negative relationship between source-monitoring scores and latencies to describe objects' functions, we computed several correlations. Within each type of picture category, a correlation was calculated between the source-monitoring scores and the latencies to describe the functions of objects portrayed in the pictures. The correlations are summarized in Table 3. In 7 of the 8 cases, there was no correlation between source-monitoring performance and latencies to describe objects' functions. And, in the one case in which the correlation was significant, the relationship was positive and involved simplified versions of complex pictures rather than complex versions of pictures. Thus, there is no evidence for a strong relationship between source monitoring and latency.

The paucity of significant correlations between source monitoring and latencies does not reflect an absence of an 
Table 3

Correlations Between Source-Monitoring Performance and Latencies in Experiment 2 Actual Rendering

\begin{tabular}{lcc}
\cline { 3 - 3 } Natural Rendering & Visually Simple & Visually Complex \\
\hline Visually simple & & \\
$\quad$ Complete pictures & .04 & .21 \\
$\quad$ Incomplete pictures & .14 & .08 \\
Visually complex & & \\
$\quad$ Complete pictures & .26 & .03 \\
Incomplete pictures & $.32^{*}$ & .03 \\
\hline
\end{tabular}

*Correlation significant with alpha at .05 .

effect of picture category on latency. An ANOVA on latencies, including completeness, natural rendering, and actual rendering as factors, revealed a main effect of actual rendering $[F(1,45)=17.68, p<.001]$, qualified by a three-way interaction $[F(1,45)=20.187, p<.001]$. Subsequent tests indicated a very different pattern for simple and complex materials, as summarized in Table 4. Specifically, for pictures typically experienced and presented as complex in their visual detail, latencies to begin to describe objects' functions were equivalent for complete and incomplete pictures. But, for complex materials actually experienced in the experiment as visually simple in detail, the time to begin to describe objects' functions was much greater for complete pictures than for incomplete ones. For pictures typically experienced and presented as simple in their visual detail, again, there was no difference in the latencies for complete and incomplete pictures. But, for pictures typically experienced as simple in detail and modified to appear visually complex in the experiment, it took much longer to begin to describe the functions for incomplete pictures than for complete ones.

Recognition. As in Experiment 1, the source-monitoring test was rescored for measures of recognition (hits and false positives).

Proportion of hits. The proportion of hits was calculated by summing the number of items within each picture category recognized as targets (whether or not their mode of presentation was remembered correctly) divided by the number of targets in that category. In an analysis including completeness (complete vs. incomplete), natural rendering (simple vs. complex), and actual rendering (simple vs. complex), there were no significant effects. The absence of effects was not a result of a ceiling effect; average performance was .85 .

False recognition of distractors. The analysis of the proportion of items misidentified as old (i.e., false positives) included only two factors: natural rendering (simple vs. complex) and type of error (i.e., falsely reporting "whole" vs. "partial"). (Because the test items were presented verbally, the actual rendering factor was not relevant here.) In this case, although the overall levels of error were low, the participants were still more likely to claim that a new item was presented as "incomplete" in form rather than "complete" $\left[F(1,45)=11.72, M S_{\mathrm{e}}=\right.$
$0.003, p<.001]$. The mean proportions were .04 and .01 , respectively.

Confidence ratings. For each judgment, the participants expressed the confidence in the accuracy of their decisions. Thus, it is possible to look at the confidence ratings for the discrimination scores as well as those for the two measures of recognition (hits and false positives). The participants were generally confident about the accuracy of their source-monitoring decisions (whether the analysis focused on discrimination scores or hits). Their average confidence was 8.3 out of 9.0. Analyses on the confidence ratings for discrimination scores and for proportion hits showed no significant effects. However, the participants' confidence did vary for decisions about distractors. When new items were misclassified as "complete pictures," the participants were more confident that they were correct ( $M=3.45$ on a 9-point scale) than when new items were misclassified as "incomplete pictures" $(M=1.85)\left[F(1,45)=10.18, M S_{\mathrm{e}}=11.71, p<.003\right]$.

\section{Discussion}

In Experiment 2, we replicated the bias to claim incomplete pictures were presented as complete, but only for pictures from the Snodgrass and Vanderwart (1980) norms portraying objects in their typical visual detail. Was this bias, in particular, and source-monitoring performance, in general, related to perceptual availability? The source-monitoring scores themselves suggest not. In this experiment, perceptual information was potentially more available when pictures were rendered as visually complex. However, source-monitoring performance for pictures presented in visually complex ways was worse than that for pictures presented in visually simple ways in only one case (i.e., when the depictions resembled the ways in which the pictures are usually experienced).

The correlational analyses also fail to support the perceptual availability hypothesis as an explanation for source-monitoring performance. In these analyses, perceptual information was potentially more available when the pictures were rendered in visually complex ways and when pictures were viewed for longer periods of time. We would therefore expect source-monitoring performance to correlate with response latencies at least under these conditions if perceptual availability contributed to the misattribution bias, but it does not. In fact, out of 16 correlations, only 4 were significant. These significant

Table 4

Average Latencies (in Milliseconds) in Experiment 2 (Describing Objects' Functions)

\begin{tabular}{lcc}
\hline & \multicolumn{2}{c}{ Actual Rendering } \\
\cline { 2 - 3 } Natural Rendering & Visually Simple & Visually Complex \\
\hline Visually simple & & \\
Complete pictures & 3,603 & 3,786 \\
Incomplete pictures & 3,438 & 4,403 \\
Visually complex & & \\
Complete pictures & 4,315 & 3,731 \\
Incomplete pictures & 3,535 & 3,646 \\
\hline
\end{tabular}


correlations were weak at best and were not systematic relative to each other. Thus, the results of Experiment 2 weaken the persuasiveness of the perceptual availability hypothesis as an explanation for the misattribution bias that we observed in Experiments 1 and $1 \mathrm{~A}$.

In an effort to understand the basis of this misattribution bias, in Experiment 3 we examined the possibility that the presence of incomplete pictorial information was sufficient to induce the bias to claim that incomplete pictures were presented complete in form. If the processes underlying object identification in the context of picturefragmentation tasks involve the activation of filling-in processes, then we should expect to observe the bias regardless of the orienting task. However, previous research on source-monitoring judgments suggests a different expectation. When asking people to name objects presented as pictures or words, Durso and Johnson (1980) reported no confusion about the way in which materials were presented. In contrast, as we have mentioned, when the same pictures and words are presented in the context of describing objects' functions, there is considerable confusion: People often claim that words were presented as pictures (Durso \& Johnson, 1980; Foley et al., 1991). Along similar lines, in the present Experiment 3, we may not find a bias to report "complete" following a naming task if requests for object identifications do not automatically elicit closure under conditions of partial viewing. We tested this possibility in Experiment 3 by asking participants to simply name the pictures that were presented in Experiment 2.

\section{EXPERIMENT 3}

\section{Method}

Participants. Forty-two undergraduates attending Skidmore College volunteered to participate and received extra course credit for their participation. Males and females were represented proportionally. None of these individuals participated in any of the other experiments reported in this series.

Materials and Procedures. The pictures, identical to those described for Experiment 2, were presented on a computer screen and remained in view until the participants pressed a button and named out loud the objects portrayed in the pictures. After a brief retention interval, during which the participants completed the same filler task used in Experiment 2, they were given a source-monitoring task.

\section{Results}

Source monitoring. An ANOVA was computed on the source-monitoring scores, including the factors completeness (complete vs. incomplete), natural rendering (simple vs. complex), and actual rendering (simple vs. complex). There were no significant effects for this analysis. Notably, the bias to claim that an incomplete picture was presented as complete in form was not evident following a naming task. The average discrimination scores were 0.86 and 0.91 for complete and incomplete pictures.

Latencies. Within each type of picture category, a Pearson product-moment correlation was calculated between the source-monitoring scores and the latencies to name the objects portrayed in the pictures, using the .05 level of significance. In 6 of the 8 cases, there was no correlation between source-monitoring performance and latencies to name. Furthermore, in the 2 cases in which the correlations were significant, neither was particularly strong, nor was the pattern consistent with the availability hypothesis (see Table 5).

The absence of strong relationships between source monitoring and latencies is not because latencies were equivalent across the picture categories. In an ANOVA on the latencies, there were several significant effects. On average, incomplete pictures were identified more slowly than were complete ones $\left[F(1,39)=24.84, M S_{\mathrm{e}}=\right.$ $705,198.72, p<.001]$. There were also main effects for natural rendering and actual rendering $[F(1,39)=$ $25.01, M S_{\mathrm{e}}=343,586.63$, and $F(1,39)=43.94, M S_{\mathrm{e}}=$ $202,122.05$, respectively; $p s<.001]$. However, these main effects were qualified by a significant three-way interaction of completeness, natural rendering, and actual rendering $\left[F(1,39)=6.75, M S_{\mathrm{e}}=230,414.74, p<.01\right]$. As shown in Table 6 and supported by post hoc tests, the time to name objects portrayed as visually complex in form was faster for complete renderings than for incomplete renderings regardless of whether the actual renderings were natural or modified (here, naturally complex rendered as simple). In contrast, the time to name objects portrayed as visually simple in form was greater for incomplete pictures than for complete ones, and this effect was less for natural renderings than for modified ones (here, naturally simple rendered as complex).

Recognition. The source-monitoring test was rescored for recognition performance (hits and false positives).

Proportion of hits. The proportion of hits was calculated by summing the number of items within each category correctly recognized as targets (whether or not their mode of presentation was remembered correctly) divided by the number of targets in that category. In an analysis including completeness (complete vs. incomplete), natural rendering (simple vs. complex), and actual rendering (simple vs. complex), there were two significant effects. Proportion of hits was higher for incomplete pictures $(M=0.86)$ than for complete ones $(M=0.76)$ $\left[F(1,39)=10.70, M S_{e}=0.07, p<.002\right]$. Proportion of hits was higher for those pictures presented as visually simple in detail $(M=0.83)$ than for those presented as visually complex $(M=0.80)$, independent of the way in

Table 5

Correlations Between Source-Monitoring Performance and Latencies in Experiment 3

\begin{tabular}{lcc} 
& \multicolumn{2}{c}{ Actual Rendering } \\
\cline { 2 - 3 } Natural Rendering & Visually Simple & Visually Complex \\
\hline Visually simple & & \\
Complete pictures & .04 & $-.35^{*}$ \\
Incomplete pictures & .20 & .04 \\
Visually complex & & \\
Complete pictures & .09 & .01 \\
Incomplete pictures & .13 & $.34^{*}$ \\
\hline
\end{tabular}

*Correlation significant with alpha at .05 . 
Table 6

Latencies (in Milliseconds) for Each Picture Category in Experiment 3 (Naming Objects)

\begin{tabular}{lcc} 
& \multicolumn{2}{c}{ Actual Rendering } \\
\cline { 2 - 3 } Natural Rendering & Visually Simple & Visually Complex \\
\hline Visually simple & & \\
Complete pictures & 898 & 1,285 \\
$\quad$ Incomplete pictures & 1,275 & 2,232 \\
Visually complex & & \\
Complete pictures & 952 & 964 \\
Incomplete pictures & 1,232 & 1,232 \\
\hline
\end{tabular}

which those pictures were naturally portrayed $[F(1,39)=$ $\left.4.08, M S_{\mathrm{e}}=0.02, p<.05\right]$.

"False recognition" of distractors. The analysis of the proportion of items misidentified as old (i.e., false positives) included only two factors: natural rendering (simple vs. complex) and type of error (i.e., falsely reporting "whole" vs. "partial"). (Because the test items were presented verbally, the actual rendering factor was not relevant here.) In this case, although the overall levels of error were low, the participants were still more likely to claim that a new item was presented as "incomplete" in form rather than "complete" $\left[F(1,39)=17.07, M S_{\mathrm{e}}=\right.$ $0.002, p<.001]$. The mean proportions were .03 and .01 , respectively.

Confidence ratings. Analyses of the confidence ratings for each of the three memory measures (source monitoring and rescoring of the source-monitoring test for hits and false positives) showed significant effects in only one case. The participants were generally confident about the accuracy of their source-monitoring decisions (whether the analysis focused on discrimination scores or hits). Their average confidence was 8.3 out of 9.0 , and there were no significant effects for either dependent measure. However, the participants' confidence did vary for distractors. When new items were misclassified as "complete pictures," the participants were more confident that they were correct ( $M=3.59$ on a 9-point scale) than when new items were misclassified as "incomplete pictures" $(M=$ 2.23) $\left[F(1,39)=7.58, M S_{\mathrm{e}}=9.76, p<.009\right]$. Furthermore, the participants were more confident about their misclassifications of distractors as "new" when the pictorial referents for these distractors were typically rendered in visually complex ways $(M=3.85)$ than when the referents were typically rendered in visually simple ways $(M=1.98)\left[F(1,39)=9.60, M S_{\mathrm{e}}=14.12, p<.003\right]$.

\section{Discussion}

The results of Experiment 3 are important for two reasons. First, this experiment shows that the mere presence of incomplete pictorial information is not sufficient to produce the bias to claim that an incomplete picture was presented complete in form. When incomplete pictures are presented in the context of a naming task (Experiment 3 ), there was no bias to claim that the pictures were experienced as complete in form. In the context of describing functions (Experiments 1, 1 A, and 2), this bias was quite pronounced. To the extent that the bias is an index of the activation of automatic imaginal processing, its absence from source-monitoring performance in Experiment 3 is intriguing in its suggestion that the mere presence of incomplete pictorial information does not guarantee the activation of closure processes. Second, the results further weaken the persuasiveness of the perceptual availability explanation for the bias that we observed in Experiments 1 and $1 \mathrm{~A}$ because, again, there was no systematic evidence for strong relationships between latency (as an index of the availability of perceptual information) and source monitoring.

In Experiment 4 we examined whether or not a similar bias is evident when faces are presented and processed under one of three kinds of orienting tasks new to this series of experiments. Typically, adults process faces configurationally - for example, abstracting configural characteristics, such as "large, wide set eyes for such a long narrow face" (Carey, Diamond, \& Woods, 1980, p. 265). We attempted to affect the way in which faces were processed by asking individuals to complete one of three kinds of orienting tasks. Specifically, we asked the participants to rate the extent to which each face reminded them of someone they knew, the extent to which each face was a distinctive one, or the extent to which each face had a distinctive feature. The first two orienting tasks were conceptualized as configural processing tasks, but the third was conceptualized as one that might induce more piecemeal processing (e.g., encouraging participants to notice things like bushy eyebrows, a mustache, or a mole). In all three orienting conditions, faces were presented in full frontal view, but, for half of the face stimuli, one side of the face was explicitly removed, analogous to the way materials were created in Experiment 1. After a brief retention interval, all participants were asked whether each of several test faces was presented previously. If they reported "yes," they were asked to remember the ways in which the faces were presented (complete vs. incomplete).

\section{EXPERIMENT 4}

\section{Method}

Participants. One-hundred twenty undergraduates participated in this experiment, receiving $\$ 2$ or extra course credit for their participation. Males and females were represented equally across conditions, as were the students from Skidmore and Union Colleges All individuals were advanced-level students, but not necessarily psychology majors. Advanced-level students were selected as participants in order to increase the likelihood that they would recognize the faces of faculty from their respective institutions.

Norming materials. Over 110 faculty members from Skidmore College and Union College provided our facial stimuli. Each faculty member was photographed standing in front of a neutral wall so that no cues were available about the institution with whom the person was affiliated or the discipline within which the individual worked. The slides depicted only faces taken in full frontal view. An equal number of male and female faculty members was depicted in the slides.

Our intention was to manipulate face familiarity by classifying Skidmore College faculty faces as familiar and Union College faculty faces as unfamiliar for individuals attending Skidmore College. 
To ensure that any effects observed were not unique to the Skidmore College community, we included a second sample of individuals, students attending Union College. Thus, Skidmore College faculty faces were classified as unfamiliar and Union College faculty faces were classified as familiar for students attending Union College.

Materials. Ninety-six faces were selected from the original set, 48 of Skidmore College faculty and 48 of Union College faculty From this set, 24 faces from each set of faculty were chosen as target faces. Half of these were presented in full view, and half were presented in partial view. Half of the faces presented in full view depicted men (6), and half presented women (6); the same was the case for the faces presented in partial view. The incomplete facial stimuli were presented so that the right or left half of the face was missing, explicitly deleted from the slide. Because the left-side composite of a face is considered a better likeness to the whole face than is the right-side composite (Rhodes, 1985), the side of face involved in the deletion was carefully controlled and counterbalanced. For the source-monitoring test, all 96 faces were presented in full view. Target and distractors were randomly presented; two test orders were used.

Procedure. Four or 5 participants were tested at a time, and the data from the two individual populations were collected during the same time period. The participants were told that we were norming a set of facial stimuli and that they would be asked to rate each of several faces. They were also told that some faces were incomplete and that our goal was to see if the ratings differed for the two kinds of faces. The participants were randomly assigned to one of the three orienting conditions, with an equal number from each institution represented in each condition. Slides of the faces were projected onto a white wall approximately $4 \mathrm{ft}$ in front of the individuals. Thus, faces appeared close to life-sized. The slides were presented at a 10 -sec rate during both the rating phase and the test phase.

Depending on the condition to which they were assigned, during the orienting tasks, the participants were asked to record (1) a resemblance rating indicating the extent to which each face reminded them of someone they knew, (2) a distinguished-face rating indicating the extent to which each face was distinctive, or (3) a feature rating indicating the extent to which each face included a distinguishing physical feature. The rating scales ranged from 1 to 9. After a 5-min retention interval, during which time the participants completed a search task involving number sequences, they were surprised with a source-monitoring task. They were asked to indicate whether each slide portrayed a face presented during the first phase, circling an option (YES or NO) on a response sheet. If they responded "yes," they were asked to indicate whether the face was presented complete or incomplete in form in the earlier part of the experiment.

\section{Results}

Source-monitoring scores, proportion of hits and proportion of false positives were the dependent variables of interest. In preliminary analyses of each of the dependent variables reported below, neither gender of participant nor gender of person portrayed in the pictures was significant; these factors were collapsed across in the analyses reported below. ${ }^{1}$ In each case, an ANOVA including orienting task (resemblance vs. distinguished face vs. distinguishing feature), type of face (familiar vs. non familiar), and completeness (complete vs. incomplete) was conducted.

Source monitoring. Source-monitoring ability was assessed with a discrimination score, the proportion based on the number of items whose presentation mode (complete vs. incomplete) was correctly identified relative to those correctly recognized as old. One individual's data were omitted from the analysis of the discrimination data because this individual failed to classify a few of the items designated as "old."

In the analysis of source-monitoring performance, one effect was significant. As in Experiment 1, the participants were more likely to claim mistakenly that an incomplete face was presented as complete in form, rather than the reverse, producing lower discrimination scores for incomplete facial stimuli $(M=0.72)$ than for complete ones $(M=0.83)\left[F(1,116)=28.69, M S_{\mathrm{e}}=0.048, p<.001\right]$. Neither the familiarity manipulation nor orienting task affected the discrimination scores, nor were any interactions significant. The mean discrimination scores for familiar and unfamiliar faces were 0.78 and 0.77 , respectively. The mean discrimination scores for the three orienting tasks were $0.78,0.75$, and 0.79 for the resemblance ratings, distinguished-face ratings, and distinguished-feature ratings, respectively.

Recognition. The average proportion of hits was lower for faces presented incomplete in form $(M=0.78)$ than for faces presented complete in form $(M=0.93)[F(1,117)$ $\left.=184.91, M S_{\mathrm{e}}=1.99, p<.001\right]$. No other effects were significant. When examining the frequency with which the participants mistakenly classified a new face as one presented in "full" or "partial" view, they did not exhibit a bias to claim "complete" if a new face was misclassified as old. Instead, the analysis on the proportion of false positives committed showed a different response bias. The participants were more likely to claim that a new face was presented in partial view $(M=0.08)$ than in complete view $(M=0.03)\left[F(1,114)=141.96, M S_{\mathrm{e}}=3.52, p<\right.$ $.001]$. The size of this difference in proportions varied for the three orienting tasks $[F(2,117)=3.68, p<.03]$. As shown in Table 7, the difference was greatest for those who rated the extent to which each face had a distinctive feature.

Experiment 4A. We conducted a second version of this experiment in which the facial stimuli were presented on a slide screen located about $2 \mathrm{ft}$ in front of the participants, creating smaller images on the screen than in Experiment 4 (more comparable in size to those presented on index cards in the first two experiments). In this case, only Skidmore College students $(n=25)$ served as participants. The misattribution biases reported above were observed in this second version as well. The participants were more likely to claim that an incomplete face was presented complete in form, producing lower discrimination scores for incomplete faces $(M=0.75)$ than for com-

Table 7

Orienting Task and Presentation

Mode Interaction in Experiment 4

\begin{tabular}{|c|c|c|c|}
\hline \multirow[b]{2}{*}{$\begin{array}{c}\text { Distractor Face } \\
\text { Misclassified As }\end{array}$} & \multicolumn{3}{|c|}{ Type of Orienting Task } \\
\hline & $\begin{array}{r}\text { Resemble } \\
\text { - Someone } \\
\end{array}$ & $\begin{array}{c}\text { Facial } \\
\text { Distinctiveness }\end{array}$ & $\begin{array}{c}\text { Feature } \\
\text { Distinctiveness }\end{array}$ \\
\hline "Complete" & .14 & .14 & .20 \\
\hline "Incomplete" & .05 & .07 & .06 \\
\hline
\end{tabular}


plete faces $(M=0.83)\left[F(1,24)=5.12, M S_{\mathrm{e}}=0.029\right.$, $p<.03]$. No other effects were significant.

\section{Discussion}

The results of Experiment 4 extend the generality of our findings by showing that the bias to claim that incomplete pictures were presented as complete in form was evident whether the referents were objects or faces. Notably, this bias was not evident in the false-positive data, suggesting that it is not a reflection of a general response bias to claim "complete." In fact, in the present experiment, when a new face was misclassified as a target face, the opposite bias was observed. If the participants misclassified a distractor as "old," they were more likely to claim falsely that it was presented in a "partial" version rather than a "complete" version. The incidence of this false-positive bias was not independent of orienting task. When falsely identifying distractors as "old faces," regardless of orienting task, the participants were more likely to report "incomplete" than "complete." This bias was most pronounced, however, for those participants who inspected faces for unusual features.

At first glance, one might expect that incomplete familiar faces would be more likely to elicit implicit imaginal processing, leading to lower source-monitoring scores. The absence of an effect for familiarity is intriguing in its suggestion about the information that is activated by the presentation of incomplete pictures, a point to which we will return later.

In all the experiments reported in this series thus far, the pictures were explicitly incomplete when presented in their partial form. It was obvious to the participants that half of each picture was deliberately removed from view. This approach of explicitly removing portions of visual information is analogous to the approach taken in other object recognition studies (e.g., Biederman, 1987; Blickle, 1989). For example, in Gollin's (1962) early experiments as well as those done more recently by Biederman and his colleagues (Biederman \& Ju, 1988; Blickle, 1989), portions of pictures were explicitly deleted. Thus, an individual might see a picture of an airplane or a horse with portions obviously deleted from view. Similarly, in McClelland and Rumelhart's (1981) work, portions of words were removed in such a way that the presentation of words was incomplete because they were covered by "blobs" designed to resemble ink spilled on words. Though the blobs were intended to function as ink spots, it was clear that the black splotches had been placed on the words intentionally. In the experiments reported thus far, our approach was similar to that of previous experiments in that we explicitly removed portions of pictures of objects or faces - specifically, one half of the pictures, all of which were presented from the frontal perspective.

In everyday life, however, when objects are viewed under conditions of incomplete visual information, the "missing" contours are often removed by occlusion. For example, an apple may actually be viewed when it is partially occluded by a book, or a person's face may be viewed with portions of the face concealed by paraphernalia (e.g.,

floppy hats, sunglasses, scarves). Whether people are confused when partial information is created implicitly by using objects to occlude other target items remains to be seen. This possibility provided the focus for Experiment 5.

\section{EXPERIMENT 5}

In Experiment 5, the incomplete forms were created in a more naturalistic and less explicit manner. In the context of a visual search task, participants were shown a complex scene in which many familiar objects were embedded (see Figure 2). Some of the pictures embedded in the scene were complete in form, whereas others were incomplete because they were partially occluded by other objects (e.g., the pictures of the horn and the eyeglasses, respectively, in Figure 2). This occlusion is more analogous to what happens when we look at objects in a real-world scene; some portions of objects are occluded by other objects that are closer to the viewer. After completing the search task, the participants were surprised with a sourcemonitoring task in which they were asked to remember

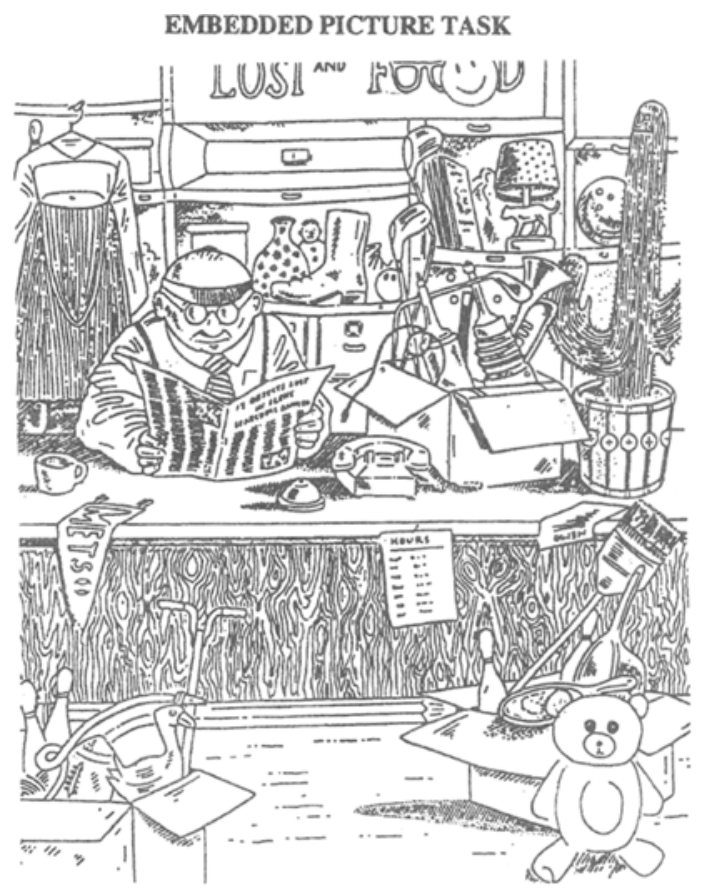

SEARCH CONDITIONS: SEARCH FOR

A HAT THIS HAT

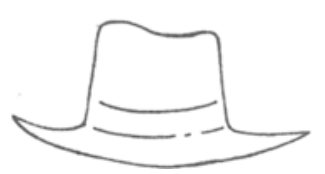

Figure 2. Embedded-pictures search task and sample prompts. Reprinted with permission from GaMEs Magazine (P.O. Box 184, Fort Washington, PA 19034). For subscription information, call 1-800-GAMES68. 
whether or not the objects they found on the search task were presented in complete or incomplete pictorial form. Our purpose was to see whether or not people were still likely to say that an incomplete picture, camouflaged in the complex scene, was presented in its complete form.

\begin{abstract}
Method
Participants. Thirty-six undergraduates attending Skidmore College volunteered, each receiving extra course credit for their participation. Males and females were represented proportionally across conditions.

Materials. The embedded-pictures task shown in Figure 2 was taken from Games Magazine. The participants searched for a total of 16 objects embedded in the complex scene in the embeddedpictures task. The original scene was altered by a student artist to ensure that each object could be presented as both complete or incomplete in form. The incomplete objects were constructed by positioning other familiar objects in front of these target items. For example, as shown in Figure 2, the participants searched for a pair of scissors (shown complete in form), and they searched for a briefcase (shown incomplete in form). Materials were counterbalanced so that the 16 items were presented complete or incomplete in form with equal frequency. Sixteen other objects served as distractors. Half of these were truly new items-that is, the participants did not search for the items, nor were the items in view in the complex scene. The other half of the distractors were portrayed in the complex scene- 4 complete and 4 incomplete in form-but the participants were not instructed to search for these items (e.g., a bowling pin).
\end{abstract}

Procedure. In all conditions, the participants were told that the purpose of the experiment was to examine individual differences in searching abilities. The participants were assigned to one of three search conditions defined by different kinds of search cues. In the first condition, they were given a list of words identifying the objects that were targets for the search task, and they were asked to search for these objects in the complex scene depicted in Figure 2. In the second condition, the participants were given pictures of the objects for which they were searching (see Figure 2). In the third condition, they were given pictures of the objects for which they were searching, but, in this case, the pictures were examples of the objects and were not identical to the forms embedded in the complex scene. In all three conditions, the participants were told that some of the objects were presented complete in form and some were partially occluded by other objects in the scene. They were also told to search until they found all of the pictures or until they were confident that they could not find any additional pictures. The participants were tested in small groups (3-5), and they were not aware that the time it took to complete the embedded-picture search task was being measured. Their success in locating embedded pictures and the time spent searching for pictures were recorded. Once the search task was completed, the participants experienced a 5-min retention interval during which they completed a number search task that served as a distractor.

Finally, the participants were surprised with a memory test that listed 32 words, 16 of which named objects included on the picture search task and 16 of which named distractor items. Again, the distractor items included 8 objects that were present in the complex scene (half complete and half incomplete in form) and 8 objects that were not displayed. For each item, the participants were asked (1) if an item was one for which they searched on the embeddedpictures task, (2) if they responded "yes," whether or not it was one they found, and (3) if they responded "yes, they found it," whether it was presented complete or incomplete in form.

\section{Results}

A number of dependent variables were analyzed for both the search task itself and the subsequent source- monitoring task. Each of these analyses included completeness (complete vs. incomplete) and type of search prompt (identical example of object, similar example, word cue) as factors.

Performance on the search task. The participants spent less time searching for objects when given prompts that were identical to the pictures on the search task $\left[F(2,33)=5.43, M S_{\mathrm{e}}=17.23, p<.01\right]$. The means for three prompt conditions were $16.08,21.00$, and $20.83 \mathrm{sec}$ for identical renditions, sample renditions, and word cues, respectively. Because time to search was measured for the entire task, rather than trial by trial, we could not compare the time spent searching for complete and incomplete pictures.

The number of objects mistakenly circled on the search task was virtually zero. The participants never mistakenly identified an object in the scene as a target (e.g., identifying the bowling pin when searching for scissors), nor did they identify some portion of the scene, thinking they had discovered a partially occluded object when they had not.

The average proportion of objects found (out of 16 ) was 74. This proportion was significantly less for incomplete pictures $(M=0.68)$ located in the embedded scene than for complete ones $(M=0.81)\left[F(1,33)=58.83, M S_{\mathrm{e}}=\right.$ $0.335, p<.001]$. The proportion found also varied with type of search cue $\left[F(2,33)=3.23, M S_{\mathrm{e}}=2.18, p<.05\right]$. Subsequent tests showed that when the prompts guiding the search were identical renditions of the target pictures, the mean proportion found (.81) was greater than in either of the other two prompt conditions (.75 for sample pictures, and .67 for word prompts). The interaction between type of picture (complete vs. incomplete) and type of prompt was not significant.

Performance on the source-monitoring task. As indicated earlier, the participants made three kinds of responses on the memory task, indicating (1) whether or not each test item was included on the search task, (2) if "yes," whether or not they found it in the scene, and (3) if "yes," whether the picture was presented complete or incomplete in form. Each of these responses is reported in turn and conditionalized on the preceding ones. Finally, we report analyses on incorrect "yes" responses to distractors.

Memory for items on the search task. Of the 16 objects included on the search task ( 8 complete and 8 incomplete objects embedded in the complex scene), on average, $93 \%$ were identified correctly as those occurring on the search task (i.e., the participants correctly responding "yes" to the first question). This proportion was equivalent for the three search conditions. The average proportion was also equivalent for objects presented complete $(M=0.93)$ or incomplete $(M=0.92)$ in form. The averages are shown in Table 8.

Memory for objects found. Given that the items were correctly recognized as part of the search task, we can next ask if individuals correctly remember whether or not they located these items on the search task (i.e., correct responses to the second question on the memory test)? As reported above, individuals found $81 \%$ of the com- 
Table 8

Proportion Recognized as Items on the Search Task in Experiment 5

\begin{tabular}{lllr}
\hline & \multicolumn{3}{c}{ Type of Prompt } \\
\cline { 2 - 3 } & Actual & & Sample \\
& Picture & Word & Picture \\
\hline
\end{tabular}

Proportion recognized as

items on search task

Complete picture

Incomplete picture

$.92 \quad 96 \quad 91$

Proportion correctly classified as

found or not (given recognized

as items on search task)

Complete picture

Incomplete picture

.99

.91

.88

Source-Monitoring Judgments: Of Those Classified

as Items Found on the Search Task, Proportion

Correctly Classified as Whole or Partial Picture

\begin{tabular}{lccc}
\hline & \multicolumn{3}{c}{ Type of Prompt } \\
\cline { 2 - 4 } \multicolumn{1}{c}{ Picture } & Actual & & Sample \\
Complete & Picture & Word & Picture \\
Incomplete & .87 & .94 & .82 \\
\hline
\end{tabular}

plete pictures hidden in the complex scene and they found $68 \%$ of the partial pictures. The measure reported here reflects the proportion of items correctly classified as found or not. Again, performance was quite good, as shown in Table 8, and there were no significant effects.

Source-monitoring judgments: Memory for the form of the embedded pictures. Our measure of source monitoring indicates the participants' ability to classify the way in which a target picture was presented (complete or incomplete in form) given that they correctly remembered finding the item on the search task. Thus, of the $81 \%$ of the complete pictures located on the search task and later correctly recognized as ones that were found, we can ask what proportion was correctly classified as "complete"? Similarly, of the $68 \%$ of incomplete items located on the search task and correctly recognized as found, what proportion was correctly classified as "incomplete"? The bias to claim that incomplete pictures were "visible" in their complete form was again observed here, and the tendency was relatively greater than in the first two experiments reported in this series, producing lower discrimination scores for incomplete pictures $(M=0.51)$ than complete ones $(M=0.88)\left[F(1,33)=59.26, M S_{\mathrm{e}}=0.07\right.$, $p<.001]$. This effect did not interact with type of prompt on the search task. The means are shown in Table 9.

Responses to distractors. When responses to the distractors were analyzed, there were no significant effects. The false-positive rate was extremely low, regardless of distractor type (i.e., picture present in the scene but not included on the search task or picture not present in the scene-a truly new item).

\section{Discussion}

The results of Experiment 5 are striking in their demonstration of a misattribution bias to claim that an incomplete picture was presented complete in its form. Thus, whether incomplete pictorial material was created explicitly by removing portions of pictures (Experiments $1,1 \mathrm{~A}, 2,4$, and $4 \mathrm{~A}$ ) or implicitly by occluding portions of objects by other objects (Experiment 5 ), this bias is evident.

\section{GENERAL DISCUSSION}

\section{Evidence for Implicit Imaginal Processing: Source-Monitoring Judgments}

Misattributions about the origin of information occur in a variety of contexts (e.g., Belli et al., 1994; Foley et al., 1991; Foley, Ratner, \& Passalacqua, 1993; Gerrig \& Prentice, 1991; Intraub \& Hoffman, 1992). As we have suggested, these asymmetric biases are interpreted as clues to the nature of representational processes. For example, cryptomnesia, or inadvertent plagiarism, occurs when individuals generate responses they believe original to them but which were expressed by someone else (Brown \& Murphy, 1989; Marsh \& Bower, 1993). These biases may indicate encoding failures in that people may fail to encode who said what if they are attending more to what they are about to say than to what another person is currently saying, leading to the false belief that they are responsible for the other person's statements (e.g., Brown \& Murphy, 1989). Or, if people covertly anticipate what another person is likely to say, they may later take credit for the utterance, forgetting that their own ideas occurred only in thought (Foley et al., 1993; Johnson et al., 1993).

In the present experiments, source-monitoring mistakes about the ways in which pictures were presented are used to make inferences about the basis of closure processes. The misclassification of incomplete pictures as complete occurred whether renderings were made incomplete by the explicit removal of pictorial detail or by occluding portions of pictures with pictures of other objects. Nevertheless, the presence of incomplete pictorial information did not guarantee the subsequent expression of this bias. When naming objects presented complete or incomplete in form, there is no subsequent bias to misclassify incomplete pictures as complete (Experiment 3 ), suggesting that implicit imaginal processing is not always evoked by incomplete pictorial information. Furthermore, when describing objects' functions, a task that did lead to this bias (Experiments 1, 1A, and 2), the bias was not observed when the pictorial renderings were different from those experienced naturally (Experiment 2, for the modified renderings). Collectively, therefore, our experiments suggest that incomplete pictures elicit imaginal completion processes involving the "filling in" of "missing" pictorial detail, but the activation of these kinds of closure processes depends on the way in which pictures are rendered and the way in which they are processed. The fact that the bias is 
not evident when the task is to simply name objects but it is evident under a variety of other orienting tasks (describing functions, searching for hidden objects, evaluating faces along several kinds of dimensions) suggests the intriguing possibility that these filling-in processes may not be operative in picture-fragmentation tasks that involve naming incomplete objects. Future work might address this possibility by using source-monitoring judgments following the completion of picture-fragmentation tasks.

\section{Evaluating Alternative Explanations For Source-Monitoring Performance}

One alternative explanation for closure described earlier uses the term closure as a referent for those conditions in which a considerable amount of perceptual information is available in a pictorial display. From this point of view, the availability of a considerable amount of perceptual information represented in a memory could falsely signal that an incomplete picture was presented complete in form. If this were the case, however, we should have observed a different set of findings. Namely, source-monitoring performance should have correlated with conditions in which the opportunity for accumulating perceptual information was relatively greater (i.e., for longer latencies and when the pictures involved visually detailed depictions). Logically, this relationship could have been negative or positive, but neither was the case.

Furthermore, from a perceptual explanation, it should also follow that there should have been a bias to report "whole" when a new item was misclassified as old, but this was not uniformly the case. In the two experiments in which a bias was observed in the false-positive data (Experiments 2 and 4), this particular bias was only observed in one case (Experiment 2). In sum, the bias to misclassify an incomplete picture as complete in form is consistent with a characterization of closure processes as the activation of implicit imaginal processing that subsequently leads to confusions about the ways in which pictures were experienced.

A second alternative interpretation of our data that makes no reference to closure proposes a tagging notion, suggesting that errors in source monitoring occur because the tag for the source of a memory (e.g., picture vs. word, picture vs. image, whole vs. partial picture) is lost or not retrieved in an all-or-none fashion (e.g., Anderson, 1983; Johnson, Foley, \& Leach, 1988). Batchelder and Reifer's (1990) alternative procedure for assessing source-monitoring judgments assumes that these sourcemonitoring judgments, and recognition processes in general, are based on a single threshold. An item is either recognized or not, and its source is either retrieved or not, implying memories are tagged for their origin. When errors in source monitoring differ for item types (in the present experiments, whole and partial pictures), if recognition of those two item types also varies in the same direction, then the errors in source monitoring may simply reflect errors in recognition. In the present experiments, however, this is not the case. We observe greater source-monitoring errors on partial pictures in several cases, despite equivalent recognition for complete and partial pictures (Experiments 1, 1A, 3, and 4).

Alternative models of recognition, in contrast, propose a continuum of "oldness," and thus a person's impression of the oldness of an item may vary. In this case, misattribution biases could reflect an impression of oldness rather than the activation of implicit imaginal processing. Confidence ratings provide an index of impressions of oldness in that individuals should express a high degree of confidence (whether correct or not) about items that appear to be quite old. If "impressions of oldness" were the basis of our effects, rather than the activation of implicit imaginal processing, we should have observed different effects in confidence ratings. For example, confidence ratings for partial pictures misclassified as whole should have been higher than confidence ratings for partial pictures correctly classified as partial, but this was not the case.

\section{Imaginal Closure Processes: \\ What Might Be Represented?}

What kinds of representations appear to result from the implicit imaginal processes activated under the conditions investigated in the present experiments? Two possibilities come to mind. The representations activated by the imaginal processing might resemble the specific versions partially represented by the incomplete pictures. When looking at an incomplete picture of a butterfly, for example, imaginal processing could involve "closing" the lines missing from the rendering in view. In this case, the imaginal processing would be tied to the specific way the pictorial information was depicted. Alternatively, the presentation of an incomplete picture of a butterfly may lead to the activation of a more generic representation for the depicted object. In the butterfly example, the presentation of an incomplete picture could lead to the imaginal experience of a "whole" image resembling a prototypical butterfly rather than a "closed" image of the particular rendering presented. Here, any rendering would potentially invoke a generic representation for the referent.

Work by Biederman and his colleagues is at least consistent with the notion that the activation processes might involve schematic representations. In a typical experiment, their participants are shown pictures of familiar objects (e.g., a complete picture of a piano) and, soon thereafter, they are shown second versions of the objects (e.g., a picture of a grand piano). When an item is preceded by itself, priming is observed (i.e., individuals quickly name the object portrayed in the second version). This priming occurs regardless of the version of the object portrayed in the prime (e.g., in the present example, the prime might be a baby piano or a grand piano; see Biederman \& Cooper, 1989). Biederman and Cooper argued that if the picture primes activated specific representations, then priming effects should be greatest when the prime and the target are visually identical, and they did not find that effect.

From our point of view, however, the presentation of incomplete pictures may indeed lead to the activation of 
more specific representations, analogous to what a completed version of the picture would look like. The fact that we observe the same misattribution bias for objects (Experiments 1, 1A, and 2) and faces (Experiment 4) is at least consistent with the view that the activation processes lead to more specific visualizations. We find the evidence from the facial stimuli in Experiment 4 particularly compelling in this regard. Because all of the stimuli in this experiment were faces, rather than a mixture of objects, it would be difficult to argue that the presentation of an incomplete face would lead to the activation of a more generic representation of that face. Furthermore, the unfamiliar faces would not have corresponding generic representations to activate, yet we observe comparable effects for familiar and unfamiliar faces. The misattribution bias was evident for both kinds of faces, arguing against the activation of more generic representations. Additional evidence for the view that the representations evoked by imaginal completion processes bear some resemblance to the specific visual information giving rise to their activation is the effects of rendering on source-monitoring judgments in Experiment 2. If the presentation of an incomplete picture leads to the activation of a more generic imaginal representation, then source-monitoring effects should have been comparable for both kinds of renderings (natural and modified), but they were not.

Directions for future work focused on the nature of the representations invoked by closure processes of the sort investigated here might include the examination of the effects on source-monitoring judgments of particular kinds of deletions (e.g., specific contours) or of particular perspectives (e.g., canonical vs. atypical). The portion of an object explicitly deleted from view affects individuals' object identification (e.g., Biederman, 1987; Blickle, 1989). If the portion removed typically expedites object recognition, then individuals might be particularly sensitive to its absence when viewing incomplete information and, hence, less likely to claim that the picture was presented complete in form. Moreover, if the kinds of contours deleted affect the magnitude of the misattribution bias, it would confirm our emphasis on the importance of the initial viewing conditions. Thus, our experiments suggest that the source-monitoring framework provides a useful heuristic for the investigation of the basis of closure processes.

\section{REFERENCES}

ANDERSON, J. R. (1983). A spreading activation theory of memory. Journal of Verbal Learning \& Verbal Behavior, 22, 262-295.

BatCheler, W. H., \& RiefER, D. M. (1990). Multinomial processing models of source monitoring. Psychological Review, 97, 548-564.

Belli, R. F., Lindsay, D. S., Gales, M. S., \& McCarthy, T. T. (1994). Memory impairment and source misattribution in postevent misinformation experiments with short retention intervals. Memory \& Cognition, 22, 40-54.

BIEDERMAN, I. (1987). Recognition-by-components: A theory of human image understanding. Psychological Review, 94, 115-147.

BIEDERMAN, I., \& COOPER, E. E. (1989). Evidence for complete translational and reflectional invariance in visual object priming. Unpublished manuscript.
Biederman, I., \& Ju, G. (1988). Surface versus edge-based determinants of visual recognition. Cognitive Psychology, 20, 38-64.

BLICKLE, T. (1989). Recognition of contour deleted images (Tech. Rep. No. 89-2). Minneapolis: University of Minnesota, Image Understanding Laboratory.

Brown, A. S., \& MuRPHY, D. R. (1989). Cryptomnesia: Delineating inadvertent plagiarism. Journal of Experimental Psychology: Learning, Memory, \& Cognition, 15, 432-442.

Carey, S., Diamond, R., \& Woods, B. (1980). The development of face recognition: A maturational component? Developmental Psychology, 16, 257-269.

DENIS, M. (1982). Imagery and prose: A critical review of the literature in adults and children. Text, 4, 381-401.

Durso, F. T., \& Johnson, M. K. (1980). The effects of orienting task on recognition, recall and modality confusion of pictures and words. Journal of Verbal Learning \& Verbal Behavior, 19, 416-429.

Foley, M. A., Durso, F. T., Wilder, A., \& Friedman, R. (1991). Developmental comparisons of the effects of explicit vs. implicit imagery generation on source monitoring. Journal of Experimental Child Psychology, 51, 1-13.

Foley, M. A., Ratner, H. H., \& Passalacqua, C. (1993). Appropriating the actions of another: Implications for children's memory and learning. Cognitive Development, 8, 373-401.

GeRrig, R. J., \& PrentiCE, D. A. (1991). The representation of fictional information. Psychological Science, 2, 336-340.

GiBson, J. (1966). The senses considered as perceptual systems. Boston: Houghton Mifflin.

Golden, C., Foley, M. A., Holtz, K., \& Lynde, R. (1994). Visualization as a guide for composing. Reading \& Writing: An Interdisciplinary Journal, 6, 197-214.

Gollin, E. S. (1962). Factors affecting the visual recognition of incomplete objects: A comparative investigation of children and adults Perceptual \& Motor Skills, 15, 583-590.

Hearst, E. (1991). Psychology and nothing. American Scientist, 79, 432-443.

HUNT, R. R., \& MARSCHARK, M. (1987). Yet another picture of imagery: The role of shared and distinctive information. In M. Pressley \& M. McDaniel (Eds.), Imagery and related mnemonic processes (pp. 129-150). New York: Springer-Verlag.

InTRAUB, H., \& HofFMaN, J. E. (1992). Reading and visual memory: Remembering scenes that were never seen. American Journal of Psychology, 105, 101-104.

Johnson, M. K., Foley, M. A., \& LeACH, K. (1988). The consequences for memory of imagining in another person's voice. Memory \& $\mathrm{Cog}$ nition, 16, 337-342.

Johnson, M. K., Foley, M. A., Suengas, A., \& Raye, C. L. (1988) Phenomenal characteristics of memories for perceived and imagined autobiographical events. Journal of Experimental Psychology: General, 117, 371-376.

Johnson, M. K., Hashtroudi, S., \& LindSay, D. S. (1993). Source monitoring. Psychological Bulletin, 114, 3-28.

Johnson, M. K., \& Raye, C. L. (1981). Source monitoring. Psychological Review, 88, 67-85.

Johnson, M. K., Raye, C. L., Foley, M. A., \& KiM, J. K. (1982). Pictures and images: Spatial and temporal information compared. Bulletin of the Psychonomic Society, 19, 23-26.

Johnson, M. K., RAYE, C. L., WANG, A., \& TAYLoR, T. (1979). Fact and fantasy: The roles of accuracy and variability in confusing imaginations with perceptual experiences. Journal of Experimental Psychology: Human Learning \& Memory, 5, 229-240.

JohnSON-LaIRD, P. N. (1983). Mental models. Cambridge, MA: Harvard University Press.

KINCHLA, R. A. (1994). Comments on Batchelder \& Riefer's multinomial model for source monitoring. Psychological Review, 101, 166-171.

LEEPER, R. (1935). A study of a neglected portion of the field of learning - The development of sensory organization. Journal of Genetic Psychology, 46, 41-75.

MARSH, R. L., \& BOWER, G. H. (1993). Eliciting cryptomnesia: Unconscious plagiarism in a puzzle task. Journal of Experimental Psychology: Learning, Memory, \& Cognition, 19, 673-688.

Matlin, M., \& Foley, H. J. (1997). Sensation and perception (4th ed.). Boston: Allyn \& Bacon. 
MCClelland, J. L., \& Rumelhart, D. E. (1981). An interactive activation model of context effects in letter perception: Part 1 . An account of basic findings. Psychological Review, 88, 375-407.

Murray, F. S., \& Kinnison, E. L. (1989). Degree of fragmentations and number of distinctive features in the recognition of pictured objects by children and adults. Bulletin of the Psychonomic Society, 27, 121-124.

Ramachandran, V. S. (1992, May). Blind spots. Scientific American, 266(5), 86-91.

RHODES, G. (1985). Lateralized processes in face recognition. British Journal of Psychology, 76, 249-271

Sekuler, A. B., \& Palmer, S. E. (1992). Perception of partly occluded objects: A microgenetic analysis. Journal of Experimental Psychology: General, 12, 95-111.

Sekuler, A. B., Palmer, S. E., \& Flynn, C. (1994). Local and global processes in visual completion. Psychological Science, 5, 260-267.

Snodgrass, J. G., \& Feenan, K. (1990). Priming effects in picture fragment completion: Support for the perceptual closure hypothesis. Journal of Experimental Psychology: General, 119, 276-296.

SNOGRass, J. G., \& Surprenant, A. (1989). Effect of retention interval on implicit and explicit memory for pictures. Bulletin of the Psychonomic Society, 27, 395-398.
Snodgrass, J. G., \& VANDERWART, M. (1980). A standardized set of 260 pictures: Norms for name agreement, image agreement, familiarity, and visual complexity. Journal of Experimental Psychology. Human Learning \& Memory, 6, 174-215.

Thurstone, L. L. (1944). A factorial study of perception. Chicago: University of Chicago Press.

\section{NOTE}

1. In this experiment, unlike the others, the test items were presented in pictorial form. This was necessary because the faculty could not be identified by name. Thus, there was a confound in that incomplete pictures were presented in a different form at the test but complete pictures were presented in the same form. However, when the discrimination scores were recomputed, analyzing an overall score combining responses to complete and incomplete facial stimuli-thus, eliminating this as a factor-the analyses were comparable to those reported above. More importantly, the nature of the bias was the same as that observed for Experiments 1 and 5, in which this confound did not occur.

(Manuscript received December 11, 1995; accepted for publication February 19, 1996.) 